\title{
Propagating magneto-hydrodynamic waves in a cooling homogenous coronal plasma
}

\author{
R. J. Morton ${ }^{1}$, A. W. Hood ${ }^{2}$, and R. Erdélyi ${ }^{1}$ \\ 1 Solar Physics and Space Plasma Research Centre (SP $\left.{ }^{2} \mathrm{RC}\right)$, University of Sheffield, Hicks Building, Hounsfield Road, \\ Sheffield S3 7RH, UK \\ e-mail: [app07rjm;robertus]@sheffield.ac.uk \\ 2 School of Mathematics and Statistics, University of St. Andrews, St Andrews, Fife KY16 9SS, UK \\ e-mail: alan@mcs.st-and.ac.uk
}

Received 28 September 2009 / Accepted 11 December 2009

\begin{abstract}
Aims. We present an investigation into how the cooling of the background plasma influences the propagation of slow and fast MHD wave modes supported by an unbounded, homogenous plasma. Previous investigations have suggested that the cooling of the plasma and a reduction in density could lead to the damping of fast magneto-acoustic oscillations. We aim to investigate whether cooling of the background plasma at a constant density may be responsible for the damping of slow and fast modes.

Methods. The plasma is assumed homogeneous and the background temperature (pressure) is decreasing with time. The temperature change is assumed to be due to optically thin radiation. A special case of the radiative function is chosen to allow an analytical assessment of the effects of cooling on magneto-acoustic MHD modes and ensures the temperature evolution of the background plasma due to this radiation also matches the observed cooling profile of coronal loops.

Results. A time-dependent dispersion relation is obtained on the slow timescale of cooling and full time-dependent solutions are found. Leading order equations for the amplitude of the waves are obtained and solved analytically for the slow and fast MHD modes. The cooling of the plasma is found to cause the frequency of the magneto-acoustic modes to decrease with time. The slow modes are found to experience a greater change in frequency than the fast modes. More importantly, the radiative losses also provide a significant damping of the slow mode and a small damping of the component of the fast mode perpendicular to the magnetic field. The damping of the slow mode is found to be strong within typical lifetimes of oscillations observed in coronal structures. Cooling could have important consequences and needs to be assessed when trying to determine what mechanism is responsible for the observed damping of coronal oscillations.
\end{abstract}

Key words. waves - magnetohydrodynamics (MHD) - Sun: corona - Sun: oscillations - plasmas

\section{Introduction}

It is well known that the solar atmosphere is a highly magnetised and dynamic plasma consisting of numerous magnetic structures (e.g. coronal loops, open flux tubes, prominences, etc.). Each of these structures is able to support a wide range of magnetohydrodynamic (MHD) waves and oscillations and are of much interest as they may provide a solution to the coronal heating problem (Erdélyi 2008; Taroyan 2008; Taroyan \& Erdélyi 2009). There have been numerous observations of oscillations within solar atmospheric magnetic structures (for reviews, see e.g. De Moortel 2005; Nakariakov \& Verwichte 2005; Banerjee et al. 2007) and the majority of them are heavily damped. In spite of considerable research efforts, the cause of damping is still unknown although numerous different mechanisms have been suggested with various degrees of success. At present, resonant absorption (Ruderman \& Roberts 2002; Goossens et al. 2002; Terradas et al. 2006) appears to be the most likely method of damping fast kink waves and thermal conduction (Ofman \& Wang 2002; De Moortel \& Hood 2003; De Moortel \& Hood 2004; Mendoza-Briceño et al. 2004) for the damping of propagating slow (longitudinal) waves and standing slow modes in hot loops $(T<6 \mathrm{MK})$. Whichever mechanisms are responsible, it is widely thought that the dissipation of the wave energy could help heat the corona.

So far, the majority of investigations into the theoretical modelling of coronal structures has focused on static solutions of the MHD equations, i.e. with time-independent background plasma quantities. This has lead to the creation of the field of coronal seismology, with a number of useful seismological tools being developed for plasma diagnostics (for the latest reviews, see, e.g. Andries et al. 2009; Ruderman \& Erdélyi 2009). One such tool is the ratio of the frequency of the fundamental mode to the first overtone of the fast kink mode, which provides estimates of the density scale height in the corona (Andries et al. 2005a). The success of this seismological tool has been due to a few observations of the fast kink mode and its overtone, see, e.g. Verwichte et al. (2004), Verth et al. (2008). A number of different authors have refined the model to include a series of parameters such as variation in the density structuring (Andries et al. 2005b; McEwan et al. 2006), loop cross section (Verth \& Erdélyi 2008; Ruderman et al. 2008), loop shape (Dymova \& Ruderman 2006; Morton \& Erdélyi 2009a).

One aspect of the dynamic atmosphere that has very recently received attention is the temperature evolution of the coronal plasma. In particular, observations have revealed that 
many coronal loops undergo an exponential temperature decrease (Aschwanden \& Terradas 2008; Ugarte-Urra et al. 2009), with characteristic cooling times of 500-2000 s reported for EUV loops. Hydrodynamic simulations suggest the decrease in temperature also appears to be associated with a decrease in the density (Jakimiec et al. 1992).

In a recent paper, Morton \& Erdélyi (2009b) suggested that standing transverse oscillations in a coronal loop would be damped due to the cooling of the plasma. No explicit cooling mechanism was stated and it was assumed that the loop was cooling due to some unspecified mechanism (e.g. radiation, thermal conduction). A density profile dependent upon the change in temperature was also taken into account. Aschwanden \& Terradas (2008) suggested at EUV temperatures $(T<2.0 \mathrm{MK})$ that radiation is the probable cooling mechanism of coronal loop plasma. Morton \& Erdélyi (2009b) also found that the cooling would cause a change in the frequency of the transverse modes, causing the frequency to increase (or the period of the mode to decrease) with time. Further, a spatial shift in the longitudinal amplitude profile of the fast kink mode also occurred providing information for spatial magneto-seismological studies.

A recent observation by Erdélyi \& Taroyan (2008) reported the observation of a standing slow mode in an EUV loop in multiple spectral lines. An increase in intensity in lower temperature lines and a decrease in higher temperature lines suggests a cooling scenario is occurring. As radiation is thought to be the dominant method of the cooling of coronal loop plasma, it may heavily influence the behavior of standing slow modes.

There have already been previous investigations into nonadiabatic plasmas and how the cooling and heating influences the oscillations supported within the plasma (Field 1965; Ibanez \& Escalona 1993; De Moortel \& Hood 2003; Terradas et al. 2005; Carbonell et al. 2006). However, the plasma background state has been time-independent in all cases and it is the applied perturbations that are being directly affected by the cooling or heating process. This approach results in an additional thermal mode being able to propagate in the medium. Ibanez \& Escalona (1993) found that wave modes (i.e. fast and slow waves) could be damped or amplified depending upon the relative sizes of various cooling/heating parameters.

Owen et al. (2009) investigated the non-linear evolution of slow waves propagating from the footpoint of a loop, through the transition region and into the corona. After analysing the damping of the wave due to thermal conduction and optically thin radiation, they identified a phase shift between the velocity, density and temperature oscillations due to thermal conduction. Next, they used forward modelling techniques to estimate the observable emission.

We present here a new investigation into the effect of a cooling background plasma state on the propagation of magnetoacoustic modes. We do not assume here a specific magnetic structure and the plasma is assumed to be uniformly magnetised. A simple, representative radiative function is chosen and the plasma then cools with an exponential time profile that is in accordance with observational estimates of the cooling profile. The density remains constant in time. A time-dependent dispersion relation is derived and the fast and slow magnetoacoustic wave modes are recovered. However, the modes now have properties, i.e. phase speed, frequency, etc. that are changing as the plasma cools. We show that radiation provides a significant damping of the perturbations of the slow modes and a small amount of damping to the component of the fast mode propagating perpendicularly to the background magnetic field.

\section{Governing equations}

Consider a homogenous plasma in which the pressure is changing as a function of time and the magnetic field is in the $z$ direction, i.e. $\boldsymbol{B}_{0}=B_{0} \hat{z}$. We assume the plasma is governed by the following MHD equations,

$$
\begin{aligned}
& \frac{\partial \rho}{\partial t}+\nabla \cdot(\rho \boldsymbol{v})=0, \\
& \rho \frac{\partial \boldsymbol{v}}{\partial t}+\rho(\boldsymbol{v} \cdot \nabla) \boldsymbol{v}=-\nabla p+\frac{1}{\mu_{0}}(\nabla \times \boldsymbol{B}) \times \boldsymbol{B}, \\
& \frac{\rho^{\gamma}}{\gamma-1}\left(\frac{\partial}{\partial t} \frac{p}{\rho^{\gamma}}+(\boldsymbol{v} \cdot \nabla) \frac{p}{\rho^{\gamma}}\right)=-\rho^{2} Q(T)+H, \\
& \frac{\partial \boldsymbol{B}}{\partial t}=\nabla \times(\boldsymbol{v} \times \boldsymbol{B}) . \\
& p=\frac{1}{\tilde{\mu}} \rho R T .
\end{aligned}
$$

Here, $R$ is the gas constant, $\tilde{\mu}$ is the mean molecular weight, $\rho^{2} Q(T)$ is the general radiation term for optically thin losses of the form and $H$ is a coronal heating term.

It has been observed that the cooling profile of coronal loops is well approximated by exponential cooling (Aschwanden \& Terradas 2008; Ugarte-Urra et al. 2009) of the form

$T=T_{0} \exp \left(-\frac{t}{\tau_{\text {cool }}}\right)$,

where $T_{0}$ is the initial temperature and $\tau_{\text {cool }}$ is the cooling time scale. To model this, consider a static equilibrium determined by a balance between radiation and heating. In the absence of thermal conduction, this equilibrium is radiatively unstable. A reduction in the coronal heating term will mean that the plasma will start to cool. The background plasma will then continue to cool in a runaway manner. To model this runaway in an approximate manner, consistent with the observations, we assume that the loss/heat terms have the form of Newton cooling. The actual choice must reflect the observed exponential cooling of the background plasma (Aschwanden \& Terradas 2008). For simplicity and as a first insight, we assume the form of $\delta p$. Hence, the background state satisfies the equation of motion, continuity, gas law and energy in the form

$$
\begin{aligned}
& \boldsymbol{v}_{0}=0, \quad \Rightarrow \quad-\nabla p_{0}=0 \\
& \frac{\partial \rho_{0}}{\partial t}=0, \quad \Rightarrow \quad \rho_{0}=\text { const. } \\
& p_{0}=\frac{1}{\tilde{\mu}} \rho_{0} R T_{0}, \\
& \frac{\partial p_{0}}{\partial t}=-\delta p_{0} .
\end{aligned}
$$

Here $\delta$ is a small quantity, so that the pressure is varying slowly with time. The precise definition of small is given below. We further assume that the background density and magnetic field are constant in time and the plasma remains static. Equation (7) has solution

$p_{0}(t)=p_{i} \exp (-\delta t)$

where $p_{i}$ is the value of pressure at $t=0$. From the ideal gas law, the temperature is also cooling exponentially in time, since the density remains constant. The evolution of the pressure is shown in Fig. 1. Comparing the exponential decay with Eq. (8) it is seen that $\delta=1 / \tau_{\text {cool }}$. The observed cooling times are $500 \mathrm{~s}<\tau_{\text {cool }}<$ $2000 \mathrm{~s}$, implying $1 / 2000 \mathrm{~s}^{-1}<\delta<1 / 500 \mathrm{~s}^{-1}$. 


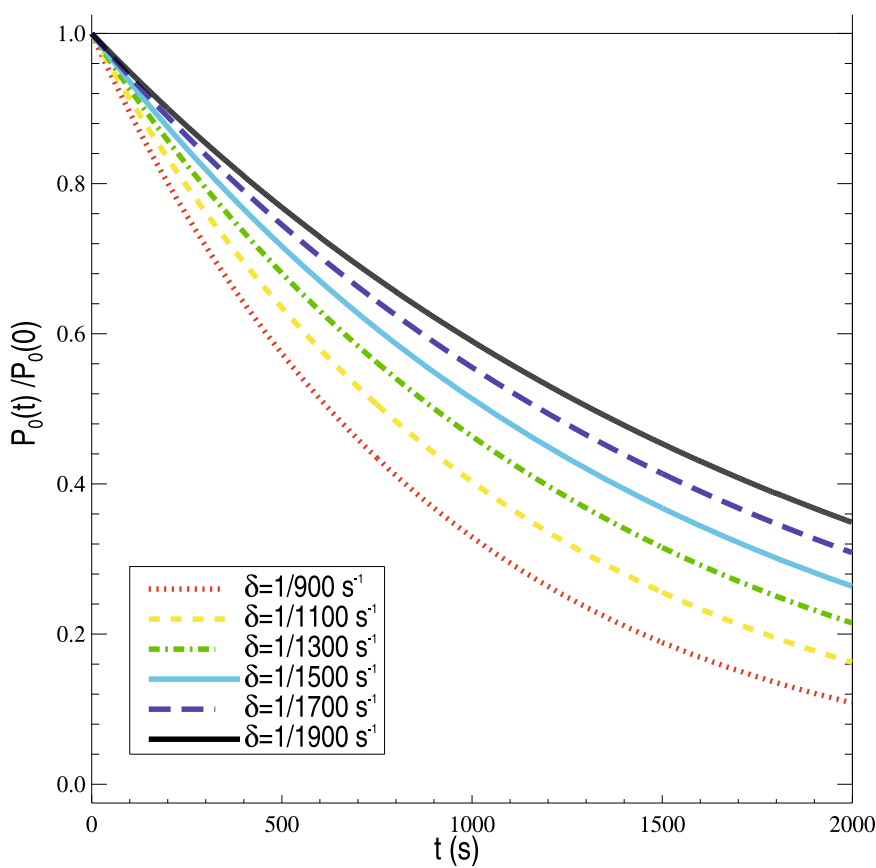

Fig. 1. Evolution of the background pressure profile of the plasma for different, characteristic values of $\delta$. The pressure is normalised with respect to the pressure at $t=0$.

We linearise the governing equations about the background state, where all variables are assumed to take the form

$f(x, z, t)=f_{0}(t)+\epsilon f_{1}(t, \boldsymbol{x})$,

where the amplitude of the perturbations, $\epsilon$, is small, i.e. $\epsilon \ll 1$. We assume that $\epsilon \ll \delta \tau<1$, i.e. $\delta \tau$ can be small or even much smaller than unity, where $\tau=L / c_{\mathrm{S}}$ is the sound speed travel time and characteristic length $L$ is the wavelength of the oscillations. At present, $\delta \tau$ is not restricted, however, it must remain larger than $\epsilon$. Assuming $\delta=1 / \tau_{\text {cool }}$, the condition $\delta \tau<1$ is satisfied for the observed cooling timescales. The linear MHD equations are given by

$\frac{\partial \rho_{1}}{\partial t}+\nabla \cdot\left(\rho_{0} \boldsymbol{v}_{1}\right)=0$,

$\rho_{0} \frac{\partial \boldsymbol{v}_{1}}{\partial t}=-\nabla p_{1}-\frac{B_{0}}{\mu}\left(\nabla B_{1 z}\right)+\frac{B_{0}}{\mu} \frac{\partial \boldsymbol{B}_{1}}{\partial z}$,

$\frac{\partial p_{1}}{\partial t}=-\gamma p_{0}\left(\nabla \cdot \boldsymbol{v}_{1}\right)-\delta p_{1}$

$\frac{\partial \boldsymbol{B}_{1}}{\partial t}=\nabla \times\left(\boldsymbol{v}_{1} \times \boldsymbol{B}_{0}\right)$.

As we are considering a homogenous medium, Fourier analysis in the $x$ and $z$ directions can be performed. Assuming that all perturbations are proportional to $\exp (\mathrm{i}(k x+l z))$ where $k$ and $l$ are the wavenumbers in the $x$ and $z$ directions, respectively, then the following equations are obtained,

$\frac{\mathrm{d} \rho_{1}}{\mathrm{~d} t}+\mathrm{i} \rho_{0}\left(k v_{x}+l v_{z}\right)=0$,

$\rho_{0} \frac{\mathrm{d} v_{x}}{\mathrm{~d} t}=-\mathrm{i} k p_{1}-\mathrm{i} k \frac{B_{0}}{\mu} B_{1 z}+\frac{B_{0}}{\mu} \mathrm{i} l B_{1 x}$,

$\rho_{0} \frac{\mathrm{d} v_{z}}{\mathrm{~d} t}=-\mathrm{i} l p_{1}$,

$\frac{\mathrm{d} p_{1}}{\mathrm{~d} t}=-\mathrm{i} \gamma p_{0} \mathrm{e}^{-\delta t}\left(k v_{x}+l v_{z}\right)-\delta p_{1}$, $\frac{\mathrm{d} B_{1 x}}{\mathrm{~d} t}=\mathrm{i} l\left(v_{x} B_{0}\right)$,

$\frac{\mathrm{d} B_{1 z}}{\mathrm{~d} t}=-\mathrm{i} k\left(v_{x} B_{0}\right)$,

$\mathrm{i} k B_{1 x}+\mathrm{i} l B_{1 z}=0$.

Taking the time derivative of Eq. (15) and substituting in the Eqs. (18) and (19), the momentum equation can be written

$\rho_{0} \frac{\mathrm{d}^{2} v_{x}}{\mathrm{~d} t^{2}}=-\mathrm{i} k \frac{\mathrm{d} p_{1}}{\mathrm{~d} t}-K^{2} \frac{B_{0}^{2}}{\mu} v_{x}$

where $K^{2}=k^{2}+l^{2}$. This equation can now be written in terms of the operator

$L=\frac{\mathrm{d}^{2}}{\mathrm{~d} t^{2}}+K^{2} v_{\mathrm{A}}^{2}$,

giving

$\mathrm{L} v_{x}=-\mathrm{i} \frac{k}{\rho_{0}} p_{1}^{\prime}$,

where $v_{\mathrm{A}}^{2}=B_{0}^{2} /\left(\rho_{0} \mu_{0}\right)$ is the constant Alfvén speed and a prime denotes a derivative with respect to $t$. The energy equation can be expressed as

$\mathrm{e}^{-\delta t} \frac{d}{\mathrm{~d} t}\left(p_{1} \mathrm{e}^{\delta t}\right)=-\mathrm{i} \gamma p_{i} \mathrm{e}^{-\delta t}\left(k v_{x}+l v_{z}\right)$

and dividing through by the exponential gives

$\frac{\mathrm{d}}{\mathrm{d} t}\left(p_{1} \mathrm{e}^{\delta t}\right)=-\mathrm{i} \gamma p_{i}\left(k v_{x}+l v_{z}\right)$.

Multiplying this new equation by the operator $L$ and differentiating with respect to time, Eqs. (16) and (23) can then be substituted into obtain an equation purely in terms of the pressure perturbation, namely

$\left[\frac{\mathrm{d}^{2}}{\mathrm{~d} t^{2}}+K^{2} v_{\mathrm{A}}^{2}\right]\left(p_{1} \mathrm{e}^{\delta t}\right)^{\prime \prime}+k^{2} c_{\mathrm{si}}^{2} p_{1}^{\prime \prime}+l^{2} c_{\mathrm{si}}^{2}\left[\frac{\mathrm{d}^{2}}{\mathrm{~d} t^{2}}+K^{2} v_{\mathrm{A}}^{2}\right] p_{1}=0$,

where $c_{\mathrm{si}}^{2}=\gamma p_{i} / \rho_{0}$ is the initial value of $c_{\mathrm{s}}^{2}$. Hence, expanding the derivatives, we have the final equation for $p_{1}(t)$

$$
\begin{gathered}
\frac{\mathrm{d}^{4} p_{1}}{\mathrm{~d} t^{4}}+4 \delta \frac{\mathrm{d}^{3} p_{1}}{\mathrm{~d} t^{3}}+\left[6 \delta^{2}+K^{2}\left(c_{\mathrm{S}}^{2}(t)+v_{\mathrm{A}}^{2}\right)\right] \frac{\mathrm{d}^{2} p_{1}}{\mathrm{~d} t^{2}}+\delta\left(4 \delta^{2}+2 K^{2} v_{\mathrm{A}}^{2}\right) \\
\times \frac{\mathrm{d} p_{1}}{\mathrm{~d} t}+\left[\delta^{4}+K^{2} v_{\mathrm{A}}^{2} \delta^{2}+K^{2} l^{2} v_{\mathrm{A}}^{2} c_{\mathrm{s}}^{2}(t)\right] p_{1}(t)=0
\end{gathered}
$$

For the special case of wave propagation parallel to the magnetic field, i.e. $k=0$, Eqs. (14)-(19) separate into the fast and slow waves. The fast wave is given by

$\rho_{0} \frac{\partial v_{x}}{\partial t}=\frac{B_{0}}{\mu} \mathrm{i} l B_{1 x}, \quad \frac{\partial B_{1 x}}{\partial t}=\mathrm{i} l\left(v_{x} B_{0}\right)$,

$\Rightarrow \quad \frac{\partial^{2} v_{x}}{\partial t^{2}}=-l^{2} v_{\mathrm{A}}^{2} v_{x}$

and the slow wave by

$\left(p_{1} \mathrm{e}^{\delta t}\right)^{\prime \prime}+l^{2} c_{\mathrm{si}}^{2} p_{1}=0$

The other parameters $\rho_{1}$ and $v_{z}$ are determined from Eqs. (14) and (16), respectively, and $B_{1 z}=0$. 


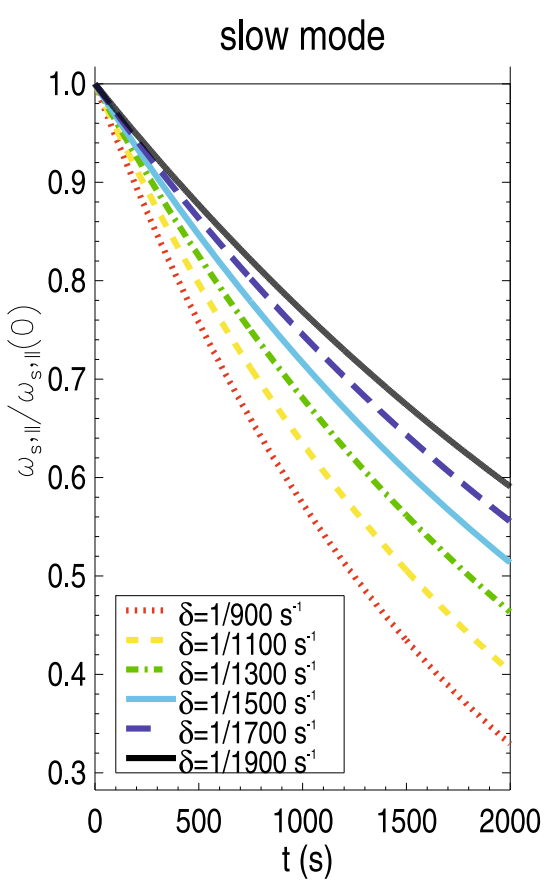

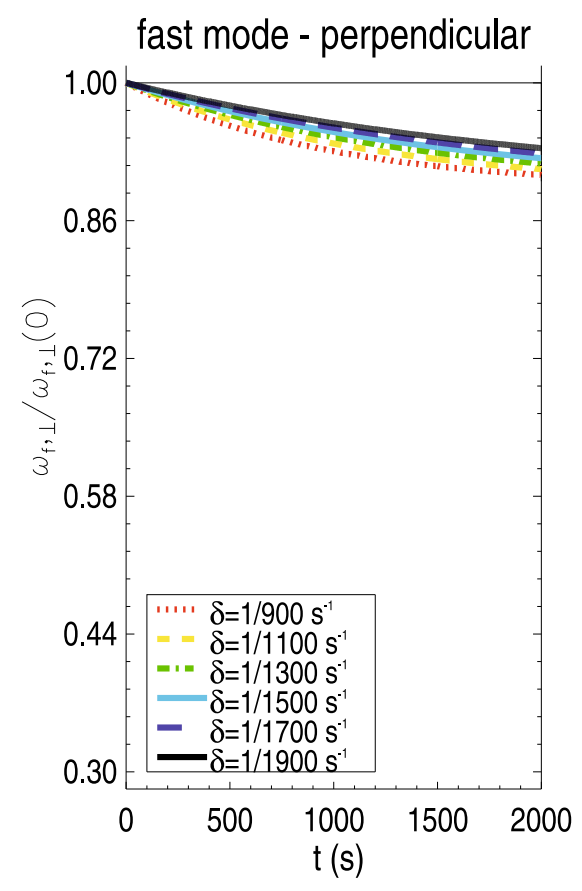

Fig. 2. Calculations of the frequency of the slow and fast modes from the analytic solutions. The frequencies are normalised with respect to the initial frequency, i.e. $\omega(t)$ at $t=0$. The wavenumbers for the slow mode are $k=0$, $K \equiv l$ since it propagates parallel to the magnetic field. For the fast mode propagating in the direction perpendicular to the magnetic field $l=0, K \equiv k$.

\section{Analytic solutions}

To understand the solution to the governing equation, (26), we shall implement WKB theory (see, e.g. Bender \& Orszag 1978) and assume that $\delta$ is small compared with the frequency of the oscillations. First, we introduce a new timescale, $t_{1}=\delta t$, namely the time over which significant cooling of the background plasma occurs. Rewriting Eq. (26) with respect to the new timescale gives

$$
\begin{aligned}
\frac{\mathrm{d}^{4} p_{1}}{\mathrm{~d} t_{1}^{4}} & +4 \frac{\mathrm{d}^{3} p_{1}}{\mathrm{~d} t_{1}^{3}}+\frac{1}{\delta^{2}}\left[6 \delta^{2}+K^{2}\left(c_{\mathrm{s}}^{2}\left(t_{1}\right)+v_{\mathrm{A}}^{2}\right)\right] \\
& \times \frac{\mathrm{d}^{2} p_{1}}{\mathrm{~d} t_{1}^{2}}+\frac{1}{\delta^{2}}\left(4 \delta^{2}+2 K^{2} v_{\mathrm{A}}^{2}\right) \frac{\mathrm{d} p_{1}}{\mathrm{~d} t_{1}} \\
& +\frac{1}{\delta^{4}}\left[\delta^{4}+K^{2} v_{\mathrm{A}}^{2} \delta^{2}+K^{2} l^{2} v_{\mathrm{A}}^{2} c_{\mathrm{s}}^{2}\left(t_{1}\right)\right] p_{1}=0 .
\end{aligned}
$$

The assumption is then made that the time-dependent part of the pressure is given by the WKB approximation

$p_{1}\left(t_{1}\right)=Q_{1}\left(t_{1}\right) \exp \left(\frac{\mathrm{i}}{\delta} \Theta_{1}\left(t_{1}\right)\right)$,

where $Q_{1}(t)$ and $\Theta_{1}(t)$ are functions to be calculated. Substituting (30) into Eq. (29) and taking the largest terms, in this case terms of order $\delta^{-4}$, we obtain

$$
\left(\frac{\mathrm{d} \Theta_{1}}{\mathrm{~d} t_{1}}\right)^{4}-K^{2}\left(c_{\mathrm{s}}^{2}\left(t_{1}\right)+v_{\mathrm{A}}^{2}\right)\left(\frac{\mathrm{d} \Theta_{1}}{\mathrm{~d} t_{1}}\right)^{2}+K^{2} l^{2} c_{\mathrm{s}}^{2}\left(t_{1}\right) v_{\mathrm{A}}^{2}=0 .
$$

Equation (31) is recognisable as the equation describing the magneto-acoustic modes (see, e.g. Lighthill 1960). However, this equation is now different as there are time-dependent terms due to the evolving pressure. The roots of the Eq. (31) are given by

$$
\begin{aligned}
\left(\frac{\mathrm{d} \Theta_{1}}{\mathrm{~d} t_{1}}\right)^{2} & =\frac{1}{2} K^{2}\left(c_{\mathrm{s}}^{2}\left(t_{1}\right)+v_{\mathrm{A}}^{2}\right) \\
& \left. \pm \frac{1}{2} \sqrt{(} K^{4}\left(c_{\mathrm{s}}^{2}\left(t_{1}\right)+v_{\mathrm{A}}^{2}\right)^{2}-4 K^{4} \cos ^{2} \theta c_{\mathrm{s}}^{2}\left(t_{1}\right) v_{\mathrm{A}}^{2}\right)
\end{aligned}
$$

which are the frequencies of the fast (positive sign) and slow (negative sign) magneto-acoustic modes. The wavenumbers have been replaced with $l=K \cos \theta$ and $k=K \sin \theta$, where $\theta$ is the angle between the magnetic field and the angle of propagation. Comparison with the time-independent equation for magneto-acoustic modes implies that $\mathrm{d} \Theta_{1} / \mathrm{d} t_{1}=\omega\left(t_{1}\right)$, i.e. is the local frequency of the modes. It is then obvious that the frequencies of the magneto-acoustic modes are changing with time as the pressure of the plasma changes. The change in frequency due the change in pressure is shown in Fig. 2 for various values of $\delta$ corresponding to representative values of observed cooling times. The solution to Eq. (32) is given by

$$
\begin{aligned}
\Theta_{1}\left(t_{1}\right) & =\int\left[\frac{1}{2} K^{2}\left(c_{\mathrm{s}}^{2}\left(t_{1}\right)+v_{\mathrm{A}}^{2}\right)\right. \\
& \left.\left. \pm \frac{1}{2} \sqrt{(} K^{4}\left(c_{\mathrm{s}}^{2}\left(t_{1}\right)+v_{\mathrm{A}}^{2}\right)^{2}-4 K^{2} l^{2} c_{\mathrm{s}}^{2}\left(t_{1}\right) v_{\mathrm{A}}^{2}\right)\right]^{1 / 2} \mathrm{~d} t_{1} .
\end{aligned}
$$

The equation of order $\delta^{-3}$ provides us with a leading order approximation of how the amplitude of the pressure perturbations evolves with time, namely

$$
\begin{aligned}
& \frac{\mathrm{d} Q_{1}}{\mathrm{~d} t_{1}}\left(2 K^{2}\left(c_{\mathrm{s}}^{2}\left(t_{1}\right)+v_{\mathrm{A}}^{2}\right) \omega-4 \omega^{3}\right) \\
& \quad+\left(K^{2}\left(c_{\mathrm{s}}^{2}\left(t_{1}\right)+v_{\mathrm{A}}^{2}\right) \omega^{\prime}+2 K^{2} v_{\mathrm{A}}^{2} \omega-4 \omega^{3}-6 \omega^{2} \omega^{\prime}\right) Q_{1}=0
\end{aligned}
$$

where the prime now indicates a derivative with respect to $t_{1}$. Once values for $\omega$ and $\Theta_{1}$ have been calculated from Eq. (32) and Eq. (33) then the values of the pressure perturbation, $Q_{1}$, can be found by solving Eq. (34). How the amplitude of pressure evolves over time is shown in Fig. 3 for a variety of modes.

Now that we have obtained a solution for the pressure perturbation, it is obvious from Eqs. (14)-(19) that one can calculate how the other perturbations, i.e. velocity, density, magnetic field, are effected by the evolving background pressure. The next step is to assume that all perturbations are of the form

$f\left(t_{1}\right)=Q\left(t_{1}\right) \exp \left(\frac{\mathrm{i}}{\delta} \int \omega \mathrm{d} t_{1}\right)$ 


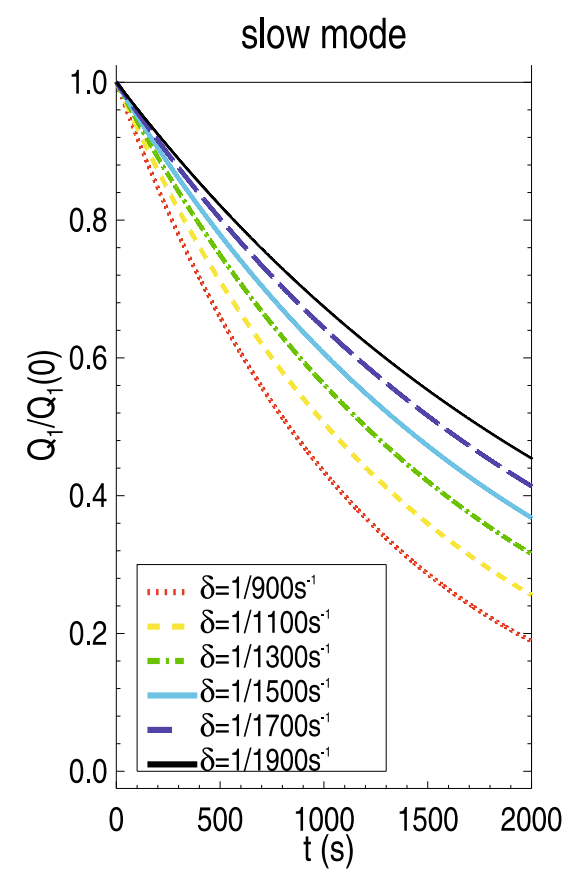

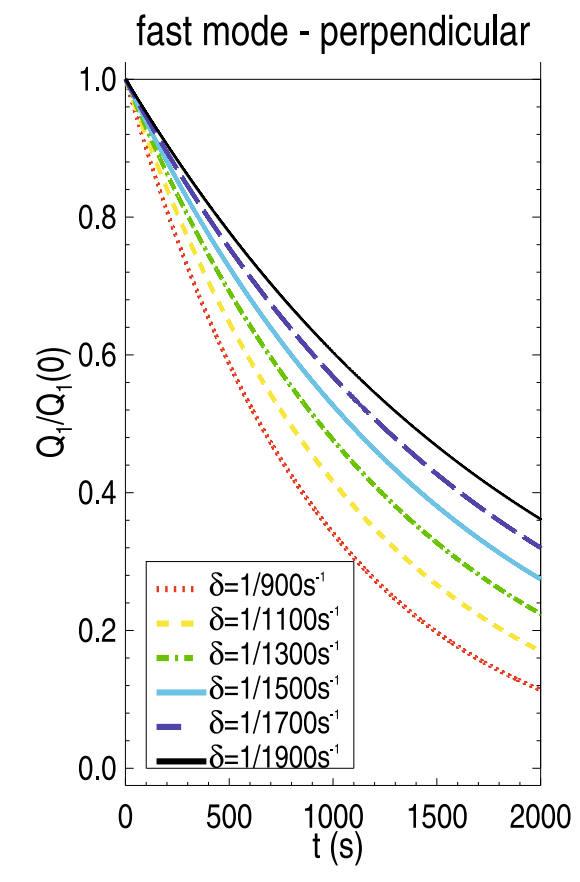

Fig. 3. Calculated values of the amplitude of the pressure perturbation for the slow and fast modes from analytic solutions. The amplitudes are normalised with respect to amplitude at $t=0$. Other parameters are the same as in Fig. 2. where $Q\left(t_{1}\right)$ is the amplitude function of each variable and $\omega$ is defined by Eq. (32).

Now, it is expected that the change in background pressure will effect the slow and fast modes to different degrees. The solutions to the governing equation for the frequency, Eq. (32), and amplitude, Eq. (34) can be applied to any plasma. We now choose to investigate the effect of a cooling plasma specific to coronal conditions, i.e. $v_{\mathrm{A}}>c_{\mathrm{S}}$ or plasma beta $<1$, on the propagation of the modes and each of the modes will be investigated individually.

\subsection{Slow mode propagation}

It is well known that the slow mode does not propagate perpendicular to the magnetic field and this can also be seen from Eq. (32) when $\theta=\pi / 2$. Now, consider slow mode propagation parallel to the magnetic field so that $k=0$ and $K \equiv l$. Oblique propagation of the slow mode is possible but the complication of the analytic solution obscures the physics. The frequency of the slow mode is given by Eq. (32) as

$\omega_{\mathrm{s}, \|}=K c_{\mathrm{s}}\left(t_{1}\right)$,

and the variation in time is shown in the left panel of Fig. 2. It is clear that the frequency of the slow mode is strongly affected by the changes in background pressure. This should not be suprising as the speed of the slow mode is strongly dependent upon the pressure of the plasma. Equation (36) can be integrated to give

$\Theta_{1}\left(t_{1}\right)=2 K c_{\mathrm{si}}\left[1-\exp \left(-t_{1} / 2\right)\right]$.

Substituting $\omega_{\mathrm{s}, \|}$ into Eq. (34), the equation for $Q_{1}\left(t_{1}\right)$ for the slow mode is given by

$\frac{\mathrm{d} Q_{1}\left(t_{1}\right)}{\mathrm{d} t_{1}}=-\frac{3}{4} Q_{1}\left(t_{1}\right)$,

which has the solution

$Q_{1}\left(t_{1}\right)=A_{1} \exp \left(-\frac{3}{4} t_{1}\right)$, where $A_{1}$ is a constant. From the leading order approximation to Eq. (16), the $v_{z}$ component of the slow mode is given by

$v_{z} \propto Q_{2}\left(t_{1}\right) \exp \left(\frac{\mathrm{i} K}{\delta} \int c_{\mathrm{s}}\left(t_{1}\right) \mathrm{d} t_{1}\right)$,

where

$Q_{2}\left(t_{1}\right)=-\frac{l}{\omega_{\mathrm{s}, \|} \rho_{0}} Q_{1}\left(t_{1}\right)$

Summarising,

$p_{1} \propto A_{1} \exp \left(-\frac{3}{4} \delta t\right) \exp \left(\mathrm{i} \frac{2 K c_{\mathrm{si}}}{\delta}\left[1-\exp \left(-t_{1} / 2\right)\right]\right)$,

$v_{z} \propto-\frac{A_{1}}{c_{\mathrm{si}} \rho_{0}} \exp \left(-\frac{1}{4} \delta t\right) \exp \left(\mathrm{i} \frac{2 K c_{\mathrm{si}}}{\delta}\left[1-\exp \left(t_{1} / 2\right)\right]\right)$.

In the left panel of Fig. 3 the calculated value of $Q_{1}\left(t_{1}\right)$ for the slow mode is shown. The amplitude of the pressure perturbation is heavily damped by the change in background pressure. The amplitude of the $v_{z}$ component is shown in the left panel of Fig. 4, which can also be seen to be damped as the background pressure changes. However, the damping is not as strong as that of the pressure perturbation, since the velocity is divided by the decreasing sound speed. However, cooling still damps the velocity perturbations by up to $40 \%$.

\subsection{Fast mode propagation}

In contrast to the slow mode, the fast mode can propagate perpendicularly to the magnetic field. We will investigate the special cases of the fast mode propagating parallel and perpendicular to the magnetic field, although oblique propagation is also valid. For propagation perpendicular to the magnetic field, $l=0$ and $K \equiv k$, and the frequency of the fast mode is given by

$\omega_{f, \perp}=K c_{f}(t)=K\left(c_{\mathrm{s}}^{2}(t)+v_{\mathrm{A}}^{2}\right)^{1 / 2}=K \sqrt{c_{\mathrm{si}}^{2} \mathrm{e}^{-\delta t}+v_{\mathrm{A}}^{2}}$.

This can be integrated exactly to give

$\Theta_{1}\left(t_{1}\right)=K\left[v_{\mathrm{A}} \log \left(\frac{c_{f}\left(t_{1}\right)+v_{\mathrm{A}}}{c_{f}\left(t_{1}\right)-v_{\mathrm{A}}}\right)-2 c_{f}\left(t_{1}\right)\right]$. 


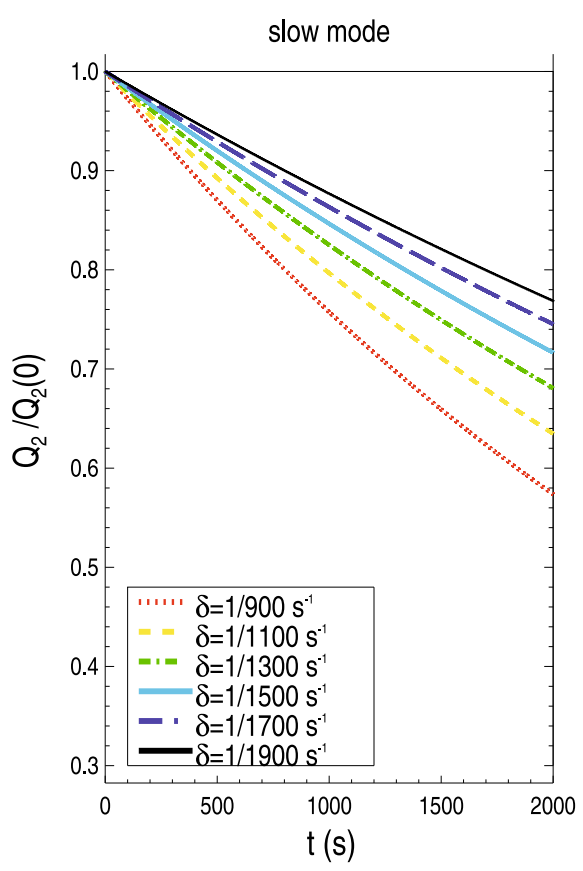

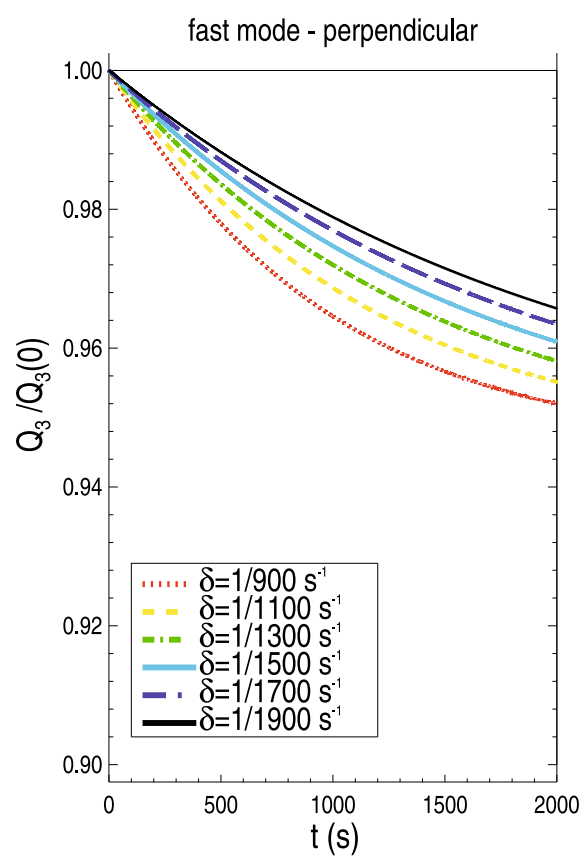

Fig. 4. Same as Fig. 3 but for amplitude of the velocity perturbations for the slow and fast modes.
The frequency, shown in the right hand panel of Fig. 2, is seen to decrease as the background pressure decreases. However, unlike the slow mode, the frequency decreases only by a relatively small amount, less than a $10 \%$ change.

An analytic solution for pressure perturbation amplitude, $Q_{1}$, for this component of the fast mode can be obtained. Substituting $\omega_{f, \perp}$ into Eq. (34), we find

$$
\begin{aligned}
& 2\left(c_{\mathrm{s}}^{2}\left(t_{1}\right)+v_{\mathrm{A}}^{2}\right)^{3 / 2} \frac{\partial Q_{1}}{\partial t_{1}}-\left(2 v_{\mathrm{A}}^{2}\left(c_{\mathrm{s}}^{2}\left(t_{1}\right)+v_{\mathrm{A}}^{2}\right)^{1 / 2}\right. \\
& \left.+\frac{5}{2}\left(c_{\mathrm{s}}^{2}\left(t_{1}\right)+v_{\mathrm{A}}^{2}\right)^{1 / 2} c_{\mathrm{s}}^{2}\left(t_{1}\right)-4\left(c_{\mathrm{s}}^{2}\left(t_{1}\right)+v_{\mathrm{A}}^{2}\right)^{3 / 2}\right) Q_{1}=0,
\end{aligned}
$$

which has the solution

$$
Q_{1}\left(t_{1}\right)=A_{2} \exp \left(-t_{1}\right)\left(c_{\mathrm{s}}^{2}\left(t_{1}\right)+v_{\mathrm{A}}^{2}\right)^{-1 / 4} \text {, }
$$

where $A_{2}$ is some constant. This solution is plotted in the right panel of Fig. 3. Maybe surprisingly, the pressure perturbation experiences a stronger damping for the fast mode than the slow mode.

The velocity component, $v_{x}$, is approximated by

$$
v_{x} \propto Q_{3}\left(t_{1}\right) \exp \left(\mathrm{i} \frac{K}{\delta}\left[v_{\mathrm{A}} \log \left(\frac{c_{f}\left(t_{1}\right)+v_{\mathrm{A}}}{c_{f}\left(t_{1}\right)-v_{\mathrm{A}}}\right)-2 c_{f}\left(t_{1}\right)\right]\right),
$$

where $Q_{3}\left(t_{1}\right)$ is a function to be determined. Substituting for $v_{x}$ and $p_{1}$ into Eq. (23), taking the leading order terms, we find the pressure and velocity perturbations for the perpendicular component of the fast mode are

$$
\begin{aligned}
p_{1} \propto & A_{2} \exp \left(-t_{1}\right)\left(c_{\mathrm{s}}^{2}\left(t_{1}\right)+v_{\mathrm{A}}^{2}\right)^{-1 / 4} \\
& \times \exp \left(\mathrm{i} \frac{K}{\delta}\left[v_{\mathrm{A}} \log \left(\frac{c_{f}\left(t_{1}\right)+v_{\mathrm{A}}}{c_{f}\left(t_{1}\right)-v_{\mathrm{A}}}\right)-2 c_{f}\left(t_{1}\right)\right]\right),
\end{aligned}
$$

and

$$
\begin{aligned}
v_{x} \propto & -\frac{A_{2} K \omega_{f, \perp}}{\rho_{0}\left(K^{2} v_{\mathrm{A}}^{2}-\omega_{f, \perp}^{2}\right)} \exp \left(-t_{1}\right)\left(c_{\mathrm{s}}^{2}\left(t_{1}\right)+v_{\mathrm{A}}^{2}\right)^{-1 / 4} \\
& \times \exp \left(\mathrm{i} \frac{K}{\delta}\left[v_{\mathrm{A}} \log \left(\frac{c_{f}\left(t_{1}\right)+v_{\mathrm{A}}}{c_{f}\left(t_{1}\right)-v_{\mathrm{A}}}\right)-2 c_{f}\left(t_{1}\right)\right]\right) .
\end{aligned}
$$

We have also plotted the amplitude of the velocity, $Q_{3}\left(t_{1}\right)$, in the right panel of Fig. 4. It can be seen that the velocity component, $v_{x}$, experiences the only a $5 \%$ decrease in amplitude in the case of strongest damping.

As above, propagation parallel to the magnetic field is when $k=0$ and $K \equiv l$. It is clearly seen from Eq. (27) that the propagation of the fast mode parallel to the magnetic field is unaffected by the change in pressure. Equation (27) does not have coefficients dependent upon $t$ so can be Fourier analysed with respect to time. Assuming the time dependence is of the form $\exp (-\mathrm{i} \omega t)$, the frequency for the fast mode parallel to the magnetic field is

$\omega_{f, \|}=K v_{\mathrm{A}}$.

This is the well known equation that is found for waves in a static plasma and is presented in many texts on plasma physics. It should be obvious from Eq. (49) that the frequency of the parallel component experiences no change due to the change in pressure. Assuming

$v_{x} \propto Q_{4}\left(t_{1}\right) \exp \left(\frac{\mathrm{i}}{\delta} \omega_{f, \|} t_{1}\right)$,

where $Q_{4}\left(t_{1}\right)$ is function to be determined. Substituting (50) into Eq. (27), it is found

$\frac{\mathrm{d} Q_{4}\left(t_{1}\right)}{\mathrm{d} t_{1}}=0$,

hence the amplitude of the velocity perturbation is constant as the pressure changes.

\section{Numerical calculations}

The WKB estimates provide simple approximations to the frequency and amplitude variations in time. However, the usefulness of these approximations can be checked by comparing the results with non-linear simulations. The aim of this section is to demonstrate how accurate these simple solutions can be. The nonlinear MHD code, Lare2d, is used to solve the MHD Eqs. (1)-(4). The code is described in detail in Arber et al. (2001). The optically thin radiative losses and coronal heating 
(a)

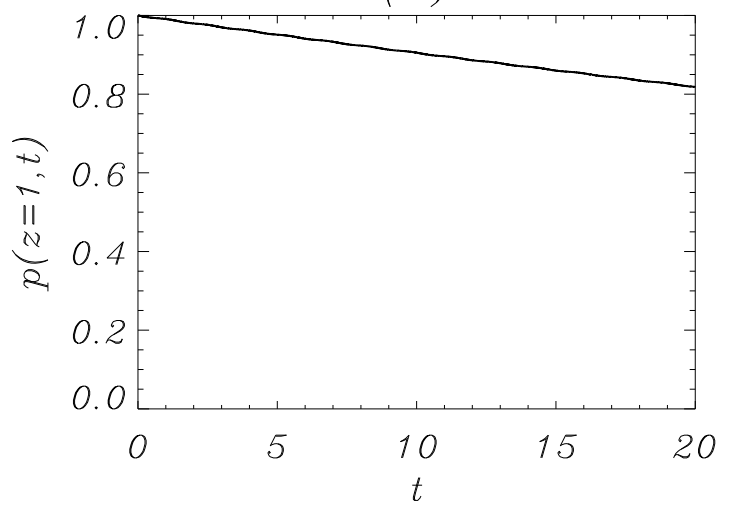

(c)

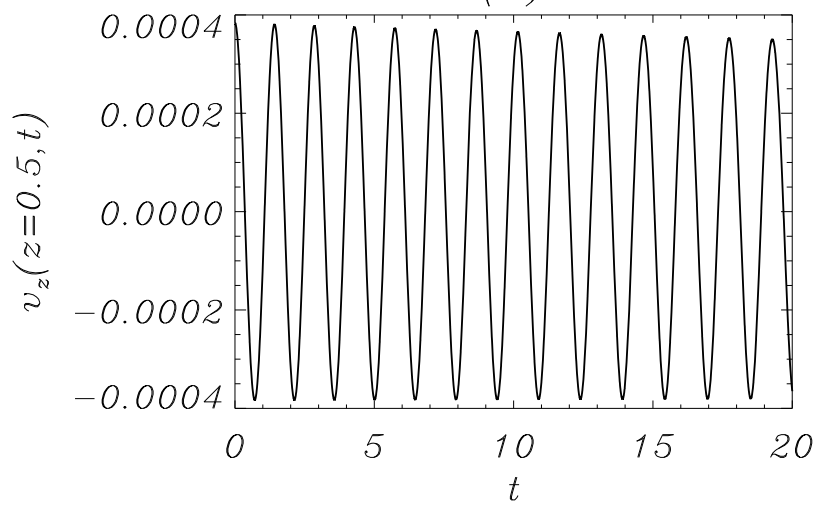

(b)

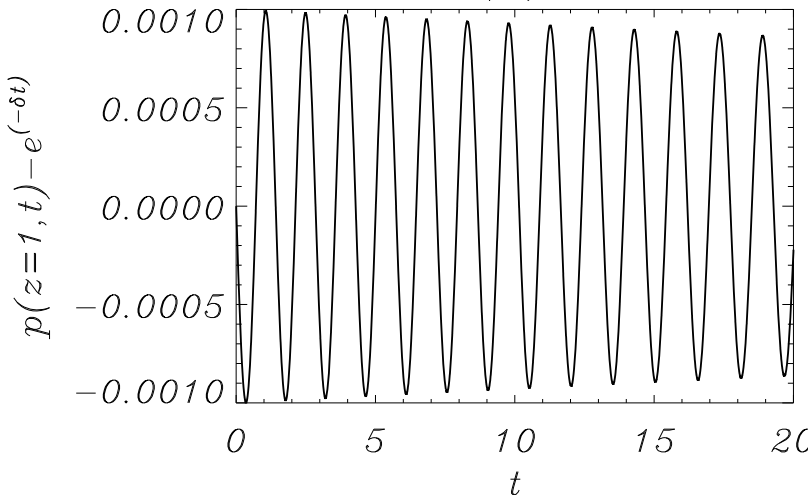

(d)

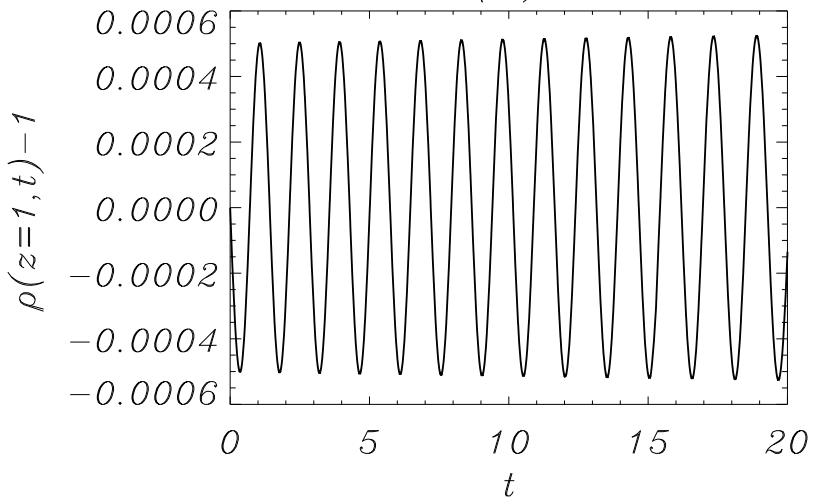

Fig. 5. Evolution of the plasma in time. a) The pressure is normalised with respect to the pressure at $t=0$. b) The pressure as a function of $t$ at $z=1$ with the background pressure, $\exp (-\delta t)$, subtracted. c) The velocity as a function of $t$ at $z=1 / 2$. d) The density as a function of time at $z=1$ with the constant background density subtracted.

are approximated by $-\delta p$ as above. An initial dimensionless velocity perturbation is given with an amplitude of $A_{1}=10^{-3}$ that will excite either the fast mode propagating perpendicular to the magnetic field, the fast mode propagating parallel to the magnetic field or the slow mode propagating parallel to the magnetic field. Obviously the linear results will be modified by nonlinear effects but these should remain small. To excite the perpendicular fast mode we select

$v_{x}=A_{1} \sin (k x)$,

and all other variables as zero at $t=0$. The fast mode parallel to the magnetic field is excited if initially

$v_{x}=A_{1} \sin (l z)$,

and the slow mode parallel to the magnetic field if

$v_{z}=A_{1} \sin (l z)$.

The results from the numerical simulation will, of course, consist of the exponentially cooling background static plasma, the linear modes and the nonlinear terms. Figure 5 shows the results of the slow wave propagating parallel to the magnetic field at specific locations in $z$ as functions of time. As the wave is only propagating parallel to the magnetic field each variable is independent of $x$. Figure 5a shows the pressure and, since the amplitude of the perturbations is small, the cooling of the background plasma is clearly seen. Figure $5 \mathrm{~b}$ illustrates the behaviour of the pressure perturbations by subtracting the background exponential decrease. The variation of the amplitude is clearly seen but the frequency change in time is not so obvious in Fig. 5b. The velocity, $v_{z}$ and the density perturbations are shown in Figs. 5c and d.

To identify the linear terms, we take a fast Fourier transform of the simulation results and select the mode consistent with the linear initial state. Thus, we can compare all the variables with the WKB solutions as functions of time. Figure 6 shows the first Fourier mode as stars and the approximate WKB solution as a solid line for (a) the pressure (where, $p_{1} \approx \exp (-3 \delta t / 4) \sin \left(\Theta\left(t_{1}\right)\right)$ ) and (b) the velocity (where $v_{z} \approx \exp (-\delta t / 4) \cos \left(\Theta\left(t_{1}\right)\right)$ ). For the slow mode, $\Theta\left(t_{1}\right)=2 l c_{\mathrm{si}}(1-\exp (-\delta t / 2)) / \delta$ and the agreement is excellent.

The fast mode propagating perpendicular to the magnetic field can be compared in a similar manner to the WKB results. Figure 7 shows the time evolution of $v_{z}(t)$ at $x=0.5$ and $z=0.5$. The numerical simulation is shown as stars (only every 30 points is plotted) while the WKB solution is shown as the solid line. Again the agreement is excellent.

\section{Discussion and conclusion}

We have investigated the effect of a cooling background on the MHD waves present in a homogenous, magnetised plasma. The plasma was assumed to be optically thin with the cooling due to radiation. However, a simplified radiative function was used. The predicted background plasma cooling profile from this function is comparable to observed cooling profiles (see, e.g. Aschwanden \& Terradas 2008). This form serves as a good 


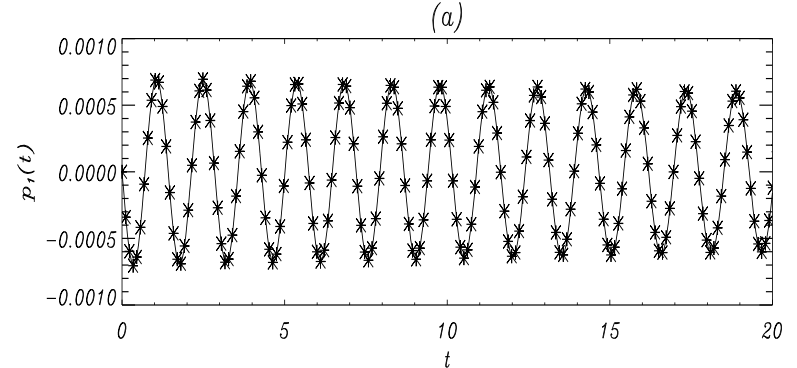

(b)

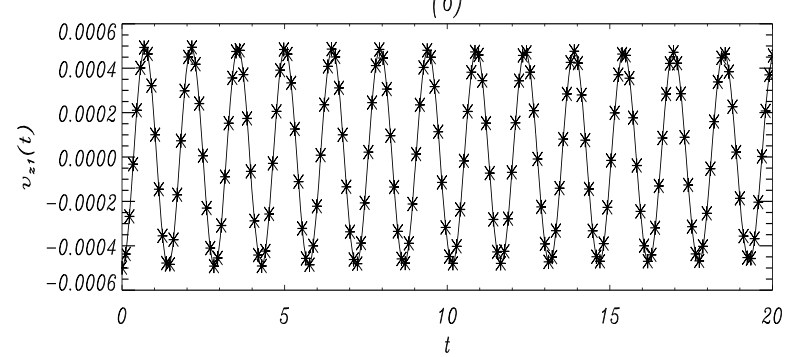

Fig. 6. The first Fourier harmonic of the numerical simulation (stars), corresponding to the imposed linear slow mode, as a function of $t$. The solid line is the appropriate WKB estimate. a) shows the Fourier component of the pressure perturbation and $\mathbf{b}$ ) the velocity perturbation.

first approximation, allowing one to gain new physical insight into oscillations in a cooling plasma. A governing equation was derived and under the assumption that the background plasma was changing on a much longer timescale than the timescale of the perturbations, a time-dependent dispersion relation for the magneto-acoustic modes was found. In previous investigations into the the effect of radiation on oscillations, background quantities have been held constant in time. It has been the effects due to the perturbation of the radiative cooling term (and/or thermal conduction term) on the wave propagation that has been examined.

The WKB method was used to solve the time-dependent governing equations. This has the limitation of only being able to provide an approximate solutions to the linear equations. The accuracy of the assumption of linearity and the WKB approximation was then checked and confirmed via numerical simulations using the non-linear Lare2D code. Excellent agreement between the analytic and numerical solutions was found, and hence, provides confidence in the analytic solutions.

For an evolving background, we find that both the slow and fast magneto-acoustic modes experience a change in frequency. The slow modes experience the greatest frequency change (left panel Fig. 2). The fast mode can be split into two separate components, one propagating parallel and one perpendicular to the magnetic field. The mode propagating perpendicular to the magnetic field experiences a change in frequency that is over a smaller range of values than the slow mode, whereas the mode propagating parallel to the magnetic field experiences no frequency change.

Aside from the frequency change, we find that a damping of the perturbations due to the cooling occurs (Figs. 3 and 4). The damping of the slow mode can be strong, both for the pressure and velocity components, with a decrease in amplitude of up to $40 \%$. This damping also occurs within observed lifetimes of the slow mode, which have typical periods of 200-1200 s in coronal structures and last up to $3600 \mathrm{~s}$ (see, e.g. Banerjee et al. 2007). This is greater than previous estimates of the damping

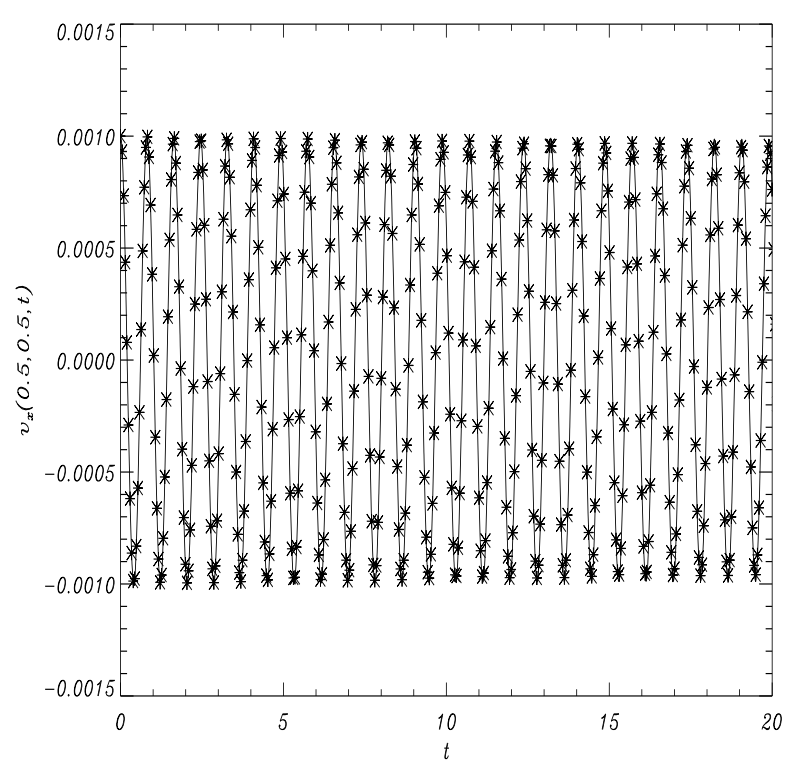

Fig. 7. The numerical simulation (stars) for $v_{z}$ at $x=0.5$ and $z=0.5$ as a function of time, corresponding to the imposed linear perpendicular fast mode, as a function of $t$. The solid curve is the WKB estimate.

of slow modes due to radiation (see, e.g. De Moortel \& Hood 2004) but a more detailed investigation using a more realistic form of optically thin radiation is needed. A combination of thermal conduction and radiation may now be able to provide a complete description of slow mode damping. However, if a similar background evolution is taken into account when solving the MHD equations with thermal conduction included, the thermal conduction could possibly be able to damp the slow modes completely by itself.

Perhaps somewhat surprisingly, the pressure perturbations of the fast mode experience the strongest damping due to the cooling. The velocity components, however, experience only a small amount of damping, with the fast mode propagating perpendicular to the magnetic field experiencing, at most, a $5 \%$ decrease in amplitude. This is most likely to be due to considering a plasma where $v_{\mathrm{A}}>c_{\mathrm{s}}$. If the plasma had a value of the plasma beta $\beta>1$, then the characteristics of the fast mode would be dominated by the plasma pressure and would probably experience a greater change in frequency and amplitude. The velocity perturbation of the fast mode propagating parallel to the magnetic field, however, is unaffected by the cooling process. The amplitude remains constant as the loop cools.

When comparing these results to those of the damping of the transverse modes in Morton \& Erdélyi (2009b), it appears that the decrease in density is mainly responsible for the calculated damping profile. Unlike the frequency, the amplitude of the kink mode is dependent upon the sound speed so there will be an effect on the amplitude from the decreasing temperature. Considering the results found for the fast wave in Sect. 3.2 this effect should be small since the value of the plasma beta in the corona is normally small $(\beta \ll 1)$. It should be noted that there is some discussion at present about the nature of the fast kink mode. Goossens et al. (2009) pointed out that the fast kink mode is highly Alfvénic and should not be described as a magnetoacoustic mode. This suggestion is particularly valid if one considers the eigenfunctions of fast kink oscillations.

This investigation shows that the physical processes responsible for the temporal evolution of the coronal plasma, i.e. the cooling of the plasma due to radiation, can also contribute to the 
damping of coronal oscillations. However, further work needs to be undertaken such as the inclusion of greater magnetic structuring, e.g. the magnetic slab or cylinder, and a more general radiative function. A similar approach can be applied to the MHD equations with the inclusion of thermal conduction terms to asses the full effect they have on the damping of slow modes. We have seen that radiation has a larger effect on slow waves than previously suggested and it may be that thermal conduction also has a greater effect on the fast waves than previously thought.

A further comment on observations of oscillations is also appropriate. We have seen that a cooling plasma can heavily influence the frequency and lifetimes of MHD oscillations. It is then necessary to establish what the observed local plasma is actually doing, i.e. is it being heated or cooled, when interpreting the oscillations.

Acknowledgements. The authors thank M. Ruderman and I. De Moortel for a number of useful discussions. R.E. acknowledges M. Kéray for patient encouragement. The authors are also grateful to NSF, Hungary (OTKA, Ref. No. K67746) and the Science and Technology Facilities Council (STFC), UK for the financial support they received.

\section{References}

Andries, J., Goossens, M., Hollweg, J. V., Arregui, I., \& Van Doorsselaere, T. 2005, A\&A, 430, 1109

Andries, J., Arregui, I., \& Goossens, M. 2005, ApJ, 624, L57

Andries, J., Van Doorsselaere, T., Roberts, B., Verth, G., Verwichte, E., \& Erdélyi, R. 2009, Space Sci. Rev., published online, DOI: $10.1007 / \mathrm{s} 11214-009-9561-2$

Arber, T. D., Longbottom, A. W., Gerrard, C. L., \& Milne, A. M. 2001, J. Comp. Phys., 171, 151

Aschwanden, M. J., \& Terradas, J. 2008, ApJ, 686, L127
Banerjee, D., Erdélyi, R., Oliver, R., \& O’Shea, E. 2007, Sol. Phys., 246, 3 Bender, C. M., \& Orszag, S. A. 1978, Advanced Mathematical Methods for Scientists and Engineers (New York: McGraw-Hill)

Carbonell, M., Terradas, J., Oliver, R., \& Ballester, J. L. 2006, A\&A, 460, 573

De Moortel, I., \& Hood, A. W. 2003, A\&A, 408, 755

De Moortel, I., \& Hood, A. W. 2004, A\&A, 415, 705

De Moortel, I. 2005, Roy. Soc. Lond. Phil. Trans. Ser. A, 363, 2743

Dymova, M. V., \& Ruderman, M. S. 2006, A\&A, 459, 241

Erdélyi, R. 2008, in Waves and Oscillations in the Solar Atmosphere, ed. B. N. Dwivedi, \& U. Narain (Princeton Series in Astrophysics), 61

Erdélyi, R., \& Taroyan, Y. 2008, A\&A, 489, L49

Field, G. B. 1965, ApJ, 142, 531

Goossens, M., Andries, J., \& Aschwanden, M. J. 2002, A\&A, 394, L39

Goossens, M., \& Terradas, J., \& Andries, J., \& Arregui, I. \& Ballester, J. L. 2009, A\&A, 503, 213

Ibanez, S. M. H., \& Escalona, T. O. B. 1993, ApJ, 415, 335

Jakimiec, J., Sylwester, B., Sylwester, J., et al. 1992, A\&A, 253, 269

Lighthill, M. J. 1960, Phil. Trans. Roy. Soc. A, 252, 397

McEwan, M. P., Donnelly, G. R., Díaz, A. J., \& Roberts, B. 2006, A\&A, 460, 893

Mendoza-Briceño, C. A., Erdélyi, R., \& Sigalotti, L. D. G. 2004, ApJ, 605, 493

Morton, R., \& Erdélyi, R. 2009a, A\&A, 502, 315

Morton, R., \& Erdélyi, R. 2009b, ApJ, 707, 750

Nakariakov, V. M., \& Verwichte, E. 2005, Living Rev. Sol. Phys., 2,3

Ofman, L., \& Wang, T. 2002, ApJ, 580, L85

Owen, N. R., De Moortel, I., \& Hood, A. W. 2009, A\&A, 494, 339

Ruderman, M. S., \& Erdélyi, R. 2009, Space Sci. Rev., published online, DOI: $10.1007 / \mathrm{s} 11214-009-9535-4$

Ruderman, M. S., \& Roberts, B. 2002, ApJ, 577, 475

Ruderman, M. S., Verth, G., \& Erdélyi, R. 2008, ApJ, 686, 694

Taroyan, Y. 2008, IAU Symp. 247, ed. R. Erdélyi, \& C. A. Mendoza-Briceño, 184

Taroyan, Y., \& Erdélyi, R. 2009, Space Sci. Rev., published online,

DOI: $10.1007 / \mathrm{s} 11214-009-9506-9$

Terradas, J., Carbonell, M., Oliver, R., \& Ballester, J. L. 2005, A\&A, 434, 741

Terradas, J., Oliver, R., \& Ballester, J. L. 2006, ApJ, 642,533

Ugarte-Urra, I., Warren, H. P., \& Brooks, D. H. 2009, ApJ, 695, 642

Verwichte, E., Nakariakov, V. M., Ofman, L., \& Deluca, E. E. 2004, Sol. Phys., 223, 77

Verth, G., \& Erdélyi, R. 2008, A\&A, 486, 1015

Verth, G., Erdélyi, R., \& Jess, D. B. 2008, ApJ, 687, L45 